\title{
Role of C5a and C5aR in doxorubicin-induced cardiomyocyte senescence
}

\author{
YAHUI WEN ${ }^{1 *}$, FEIYAN SHEN ${ }^{2 *}$ and HAIBIN WU ${ }^{3}$ \\ ${ }^{1}$ Medical Care Ward, Beijing Luhe Hospital, Capital Medical University, Beijing 101100; ${ }^{2}$ Department of Cardiology, \\ QingPu District Central Hospital, Shanghai 201700; ${ }^{3}$ Department of Outpatients, Shenzhen Traditional Chinese \\ Medicine Hospital, Guangdong Shenzhen Health Management Center, Shenzhen, Guangdong 518033, P.R. China
}

Received December 11, 2020; Accepted May 11, 2021

DOI: $10.3892 /$ etm.2021.10548

\begin{abstract}
Doxorubicin (DOX) is an efficacious antineoplastic drug; however, its use is limited due to its cardiotoxicity. Cardiomyocyte senescence is considered to be a key factor in the development of DOX-related cardiomyopathy. Complement component 5a (C5a) and the C5a receptor (C5aR) have been reported to play a key role in the process of cellular senescence. However, to the best of our knowledge, the exact role of C5a and $\mathrm{C} 5 \mathrm{aR}$ in cellular senescence in the heart remains largely unknown. Reverse transcription-quantitative (RT-q)PCR and western blot assays were used to analyze the expression levels of C5a and C5aR in H9c2 embryonic rat cardiomyocytes and AC16 human cardiomyocyte-like cells. The cells were treated with DOX and a C5aR antagonist (C5aRA). The expression of TNF- $\alpha$ and IFN- $\gamma$ was determined using ELISA and western blotting. The levels of reactive oxygen species (ROS) were also measured using ELISA. Cellular senescence was determined using senescence-associated $\beta$-galactosidase (SA- $\beta$-gal) staining and by analyzing the protein expression levels of p53, p16, p21 and insulin-like growth factor-binding protein 3 (IGFBP3). The expression levels of C5a and C5aR were found to be upregulated during the DOX-induced senescence of $\mathrm{H} 9 \mathrm{c} 2$ and AC16 cardiomyocytes. Treatment with C5aRA downregulated TNF- $\alpha$ and IFN- $\gamma$ expression, in addition to ROS levels. Furthermore, C5aRA prevented DOX-induced cellular senescence and decreased the levels of positive SA- $\beta$-gal staining in $\mathrm{H} 9 \mathrm{c} 2$ and $\mathrm{AC} 16$ cardiomyocytes, in addition to downregulating the expression levels of p53, p16, p21 and IGFBP3. C5aRA
\end{abstract}

Correspondence to: Dr Haibin Wu, Department of Outpatients, Shenzhen Traditional Chinese Medicine Hospital, Guangdong Shenzhen Health Management Center, 1 Fuhua Road, Futian, Shenzhen, Guangdong 518033, P.R. China

E-mail:wuhaibinwhb@126.com

*Contributed equally

Key words: complement component 5a, complement component $5 \mathrm{a}$ receptor, doxorubicin, complement component 5a receptor antagonist, cardiomyocyte senescence also increased the telomere length and telomerase activity in $\mathrm{H} 9 \mathrm{c} 2$ and AC16 cardiomyocytes following DOX stimulation. In conclusion, the findings of the present study indicated that C5a and C5aR may play a key role in cardiomyocyte senescence, and treatment with C5aRA may be an effective method for preventing DOX-induced cardiomyocyte aging.

\section{Introduction}

Cardiovascular disease is currently the leading cause of mortality in humans (1). Cellular senescence is defined as a state of cell cycle arrest that has the potential to promote tissue remodeling, and is involved in developmental and injury responses (2). Cellular senescence decreases tissue regenerative capacity and function, and induces inflammation and pathological remodeling of aged organs (3). Cardiomyocytes are particularly vulnerable to oxidative damage due to the lack of biochemical reserves required for successful antioxidant action (4).

Doxorubicin (DOX) is one of the most widely and successfully used antitumor agents in the clinical setting; however, its cumulative and dose-dependent cardiotoxicity has been a major concern over the previous decades and limits its use as a therapeutic agent (5). Despite the progress made over the past few decades in elucidating DOX-induced cardiomyopathy, the underlying mechanisms remain poorly understood. Oxidative stress, inflammation and apoptosis were found to be responsible for the induction of cardiotoxicity by DOX and contributed to increased levels of cardiomyocyte senescence, which led, at least in part, to heart remodeling and impairment of cardiac function (6). Therefore, identifying more specific strategies for protect patients against DOX-induced cardiotoxicity remains a priority. DOX-induced oxidative stress in $\mathrm{H} 9 \mathrm{c} 2$ cardiomyocytes represents a senescent phenotype similar to the cardiomyocyte characteristics observed in senescent rats (7).

The complement system is a central component that participates in the activation of innate immunity, inflammation and tissue remodeling. The complement activation product complement component 5a (C5a) is a potent chemoattractant involved in recruiting inflammatory cells, such as neutrophils, eosinophils, monocytes and $\mathrm{T}$ lymphocytes, inactivating phagocytes, promoting the release of granulocytes and generating oxidants (8). C5a can bind to the $\mathrm{C} 5 \mathrm{a}$ receptor (C5aR), which has been reported to be an essential modulator 
of the inflammatory response (9). The C5a/C5aR signaling pathway can activate inflammatory nuclear factor- $\kappa \mathrm{B}$, which may lead to the direct release of proinflammatory cytokines and chemokines $(10,11)$. C5aR signaling was discovered to play a key role in certain inflammation-related diseases, including acute kidney injury (12), adipose tissue inflammation (13) and cardiovascular fibrosis (14), by inducing inflammatory responses and increasing the release of inflammation-associated cytokines. The C5a/C5aR signaling pathway was also demonstrated to play an important role as a mediator of a wide range of other inflammation-related diseases, such as spinal cord injury (15), asthma (16) and myocardial ischemic injury (17). However, the role of C5a and C5aR in DOX-induced cardiomyocyte senescence remains unclear; therefore, the present study was undertaken to investigate the effects of C5a and C5aR on DOX-induced cardiomyocyte senescence.

\section{Materials and methods}

Cell culture and treatments. The $\mathrm{H} 9 \mathrm{c} 2$ rat embryonic cardiac cell line and AC16 human cardiomyocyte-like cells were purchased from The Cell Bank of Type Culture Collection of The Chinese Academy of Sciences. The cells were cultured in DMEM supplemented with 10\% FBS (both from Sigma-Aldrich; Merck $\mathrm{KGaA}$ ) and $100 \mathrm{mg} / \mathrm{ml}$ penicillin/streptomycin, and maintained in a humidified atmosphere of $5 \% \mathrm{CO}_{2}$ at $37^{\circ} \mathrm{C}$.

DOX (Sangon Biotech Co., Ltd.) was dissolved in DMSO and diluted with cell culture serum-free medium (Sigma-Aldrich; Merck $\mathrm{KGaA}$ ) to achieve a final concentration of $0.1 \mu \mathrm{M}(18)$. The C5aR antagonist (C5aRA; Shanghai Jier Biochemistry Inc.) was dissolved in DMSO and diluted with cell culture serum-free medium (Sigma-Aldrich; Merck KGaA) to a final concentration of $2.5 \mu \mathrm{g} / \mathrm{ml}$.

Reverse transcription-quantitative $(R T-q) P C R$ analysis. Total RNA was extracted from H9c2 and AC16 cells using a RNeasy Fibrous Tissue Mini kit (Qiagen, Inc.). Total RNA (1 $\mu \mathrm{g})$ was reverse-transcribed into cDNA using random hexamers and SuperScript III Reverse Transcriptase (Invitrogen; Thermo Fisher Scientific, Inc.) under the manufacturer's protocol. qPCR was subsequently performed on a LightCycler ${ }^{\circledR}$ instrument (Roche Diagnostics) using a QuantiTect SYBR-Green PCR kit (Qiagen,Inc.) by pre-denaturation at $95^{\circ} \mathrm{C}$ for $3 \mathrm{~min}$, denaturation at $95^{\circ} \mathrm{C}$ for 40 cycles of $15 \mathrm{sec}$, annealing at $58^{\circ} \mathrm{C}$ for $1 \mathrm{~min}$ and extension at $72^{\circ} \mathrm{C}$ for $30 \mathrm{sec}$. Relative gene expression levels were quantified using the $2^{-\Delta \Delta \mathrm{Cq}}$ method (19) and were normalized to GAPDH. The following primers were used for the qPCR: C5a forward, 5'-ATTGGGAAGGCTACACATGA-3' and reverse, 5'-TGCCTTGACAGTATCAGCAA-3'; C5aR forward, 5'-GAG CCCAGGAGACCAGAACATG-3' and reverse, 5'-TACATG TTGAGCAGGATGAGGGA-3'; and GAPDH forward, 5'-TGT GTCCGTCGTGGATCTGA-3' and reverse, 5'-CCTGCTTCA CCACCTTCTTGA-3'.

Western blotting. Cells were digested with trypsin and total protein was extracted from cells using RIPA lysis buffer (Beyotime Institute of Biotechnology). The protein concentration was measured with a bicinchoninic acid protein (BCA) assay kit (Beyotime Institute of Biotechnology). Total protein was adjusted to a concentration of $1 \mathrm{mg} / \mathrm{ml}$ and $10 \mu \mathrm{l}$ protein/lane was separated via SDS-PAGE on $12 \%$ gel for $90 \mathrm{~min}$, then transferred to PVDF membranes for $90 \mathrm{~min}$. The membranes were subsequently blocked with $5 \%$ skimmed milk for $2 \mathrm{~h}$ at room temperature, and after washing thrice with Tris-buffered saline Tween-20 (0.1\%, TBST), the membranes were incubated with the following antibodies at $4^{\circ} \mathrm{C}$ overnight: Anti-C5a (1:1,000; cat. no. sc-52636; Santa Cruz Biotechnology, Inc.), anti-C5aR (1:1,000; cat. no. sc-53797; Santa Cruz Biotechnology, Inc.), anti-p53 (1:1,000; cat. no. ab26; Abcam), anti-p21 (1:1,000; cat. no. ab109520; Abcam), anti-p16 (1:1,000; cat. no. ab51243; Abcam), anti-insulin-like growth factor-binding protein 3 (IGFBP3; 1:1,000; cat. no. ab220429; Abcam), anti-IFN- $\gamma$ (1:1,000; cat. no. sc-12755; Santa Cruz Biotechnology, Inc.), anti-TNF- $\alpha$ (1:1,000; cat. no. sc-12744; Santa Cruz Biotechnology, Inc.) and anti-GAPDH (1:1,000; cat. no. sc-47724; Santa Cruz Biotechnology, Inc.). Following incubation with the primary antibody, the membranes were washed thrice with TBST and incubated with horseradish peroxidase-conjugated anti-mouse IgG (1:2,000; cat. no. 7076; Cell Signaling Technology, Inc.) or anti-rabbit IgG (1:2,000; cat. no. 7074; Cell Signaling Technology, Inc.) secondary antibodies for $1 \mathrm{~h}$ at room temperature. Protein bands were visualized using a FluorChem E system (Hot Technology Co., Ltd.) after the membranes were washed thrice with TBST. The protein bands were visualized by luminescent reagents (Santa Cruz Biotechnology, Inc.) and analyzed using ImageJ software (version 1.43; National Institutes of Health).

Reactive oxygen species (ROS) assay. ROS levels were analyzed using a ROS Assay kit (Beyotime Institute of Biotechnology), according to the manufacturer's protocol. Briefly, a total of $\mathrm{H} 9 \mathrm{c} 2$ or AC16 1×10 4 cells/well were seeded into 24-well sterile culture plates and treated with $0.1 \mu \mathrm{M}$ DOX and/or $2.5 \mu \mathrm{g} / \mathrm{ml} \mathrm{C5aRA}$ for $24 \mathrm{~h}$ at $37^{\circ} \mathrm{C}$. Cells were subsequently washed thrice with phosphate buffered saline (PBS; cat no. C0221A; Beyotime Institute of Biotechnology) to remove any drug that did not enter the cells. Finally, a total of $1 \times 10^{3}$ cells per three fields of view were imaged using a fluorescence microscope under x20 magnification.

ELISA. The concentrations of TNF- $\alpha$ and IFN- $\gamma$ secreted into the cell culture media were measured using ELISA kits (cat nos. PT518 and PI511; Beyotime Institute of Biotechnology), according to the manufacturer's protocols. The absorbance was measured at a wavelength of $450 \mathrm{~nm}$ on a FlexStation 3 Multi-Mode microplate reader (Molecular Devices, LLC). Experiments were repeated in triplicate.

Senescence-associated $\beta$-galactosidase (SA- $\beta$-Gal) staining. SA- $\beta-$ Gal staining was performed using a SA- $\beta$-Gal Staining kit (cat no. C0602; Beyotime Institute of Biotechnology) according to the manufacturer's protocol. Briefly, H9c2 and AC16 cardiomyocytes were fixed with $4 \%$ formaldehyde at room temperature for $15 \mathrm{~min}$ and then washed three times with PBS for 3 mins each time. Subsequently, the cells were incubated with freshly prepared SA- $\beta$-gal staining solution overnight at $37^{\circ} \mathrm{C}$ without $\mathrm{CO}_{2}$. Stained cells were visualized using an inverted microscope (magnification, x200; Olympus Corporation). 
H9C2
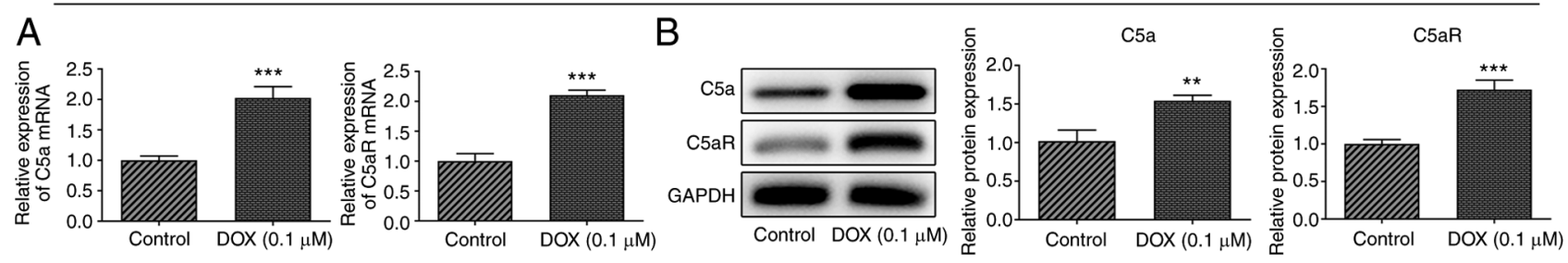

AC16
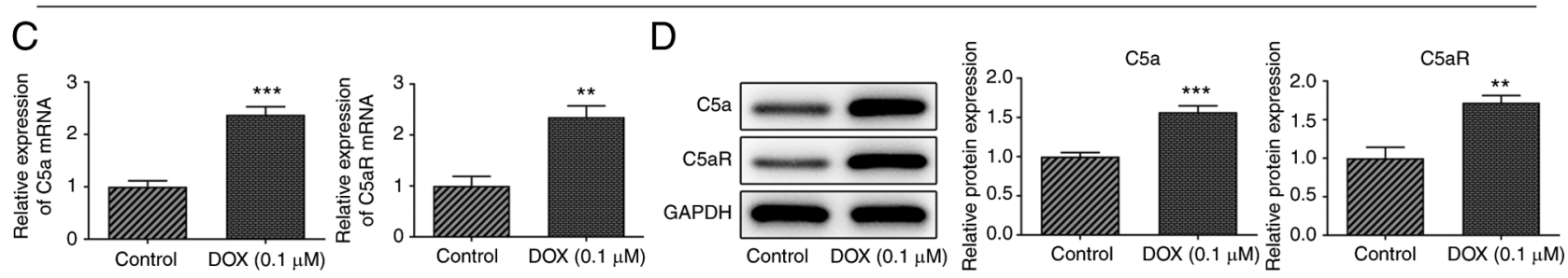

Figure 1. C5a and C5aR expression levels are upregulated in DOX-induced cardiomyocytes. Analysis of C5a and C5aR (A) mRNA and (B) protein expression levels in $\mathrm{H} 9 \mathrm{c} 2$ cardiomyocytes with or without $0.1 \mu \mathrm{M}$ DOX treatment. Analysis of C5a and C5aR (C) mRNA and (D) protein expression levels in AC16 cardiomyocytes with or without $0.1 \mu \mathrm{M}$ DOX treatment. The results are representative of at least three independent experiments. ${ }^{* *} \mathrm{P}<0.01$ and ${ }^{* * *} \mathrm{P}<0.001$ vs. control group. C5a, complement component 5a; C5aR, complement component 5a receptor; DOX, doxorubicin.

Measurement of relative telomere length. Quantification of the relevant telomere lengths in $\mathrm{H} 9 \mathrm{c} 2$ and $\mathrm{AC} 16$ cardiomyocytes was performed using qPCR according to a previously described method (20). GAPDH was used as the control gene for normalization. The primer pairs used to measure the telomere length were as follows: Forward 5'-GGTTTTTGAGGGTGA GGGTGAGGGTGAGGGTGA-3' and reverse 5'-TCCCGACTA TCCCTATCCCTATCCCTATCCCTATCC-3'.

Measurement of relative telomerase activity. The telomerase activity in $\mathrm{H} 9 \mathrm{c} 2$ and $\mathrm{AC} 16$ cardiomyocytes was analyzed using the TeloTAGGG ${ }^{\mathrm{TM}}$ Telomerase PCR ELISA PLUS kit (cat no. 11854666910; Roche Applied Science), according to the manufacturer's protocol. Briefly, cell lysates were centrifuged at $4^{\circ} \mathrm{C}$ and $12,000 \times \mathrm{g}$ for $20 \mathrm{~min}$, then $3 \mu \mathrm{l}$ inactivated cell lysate was used to amplify each telomere replicate. Inactivated cell lysates were used for the telomeric repeat amplification protocol (TRAP) reaction, according to the manufacturer's protocol (Roche Applied Science). An internal control from the kit was used along with each TRAP reaction to verify the lack of the presence of any PCR inhibitors. Amplification products were fixed in microtiter plates labeled with streptavidin via biotin-streptavidin interactions, and ELISA was performed by incubation with peroxidase-conjugated anti-dioxin antibodies for $30 \mathrm{~min}$ at $25^{\circ} \mathrm{C}$ (100 $\mu \mathrm{l}$ working solution; provided in the kit). After addition of the peroxidase substrate (3,3',5,5'-tetramethylbenzidine), the amount of TRAP product was determined by measuring the absorbance at a wavelength of $450 \mathrm{~nm}$ using a microplate reader.

Statistical analysis. Data are presented as the mean \pm standard deviation from three independent experiments. Statistical differences between more than two groups were analyzed using one-way ANOVA followed by a Tukey's multiple comparisons test. Comparisons between two groups were performed using a paired Student's t-test. $\mathrm{P}<0.05$ was considered to indicate a statistically significant difference. Statistical analysis was performed using GraphPad Prism 6.0 software (GraphPad Software, Inc.).

\section{Results}

C5a and C5aR expression levels are upregulated in DOX-induced H9c2 and AC16 cardiomyocytes. The expression levels of $\mathrm{C} 5 \mathrm{a}$ and $\mathrm{C} 5 \mathrm{aR}$ in $\mathrm{H} 9 \mathrm{c} 2$ rat embryonic cardiomyocytes and AC16 human cardiomyocyte-like cells following exposure to $0.1 \mu \mathrm{mol} / 1 \mathrm{DOX}$ were analyzed using RT-qPCR analysis and western blotting. The results revealed that DOX treatment significantly upregulated the mRNA and protein expression levels of $\mathrm{C} 5 \mathrm{a}$ and $\mathrm{C} 5 \mathrm{aR}$ in both $\mathrm{H} 9 \mathrm{c} 2$ and $\mathrm{AC} 16$ cardiomyocytes (Fig. 1). These results indicated that C5a and C5aR may play a role in DOX-induced cardiomyocytes.

Ca5RA inhibits the DOX-induced upregulation of TNF- $\alpha$ and IFN- $\gamma$ expression and increases ROS levels in $\mathrm{H} 9 \mathrm{c} 2$ and ACl6 cardiomyocytes. To determine the function of C5a and C5aR in DOX-induced cardiomyocytes, H9c2 and AC16 cardiomyocytes were treated with C5aRA and DOX. The protein and mRNA expression levels of C5a and C5aR were downregulated following co-treatment with C5aRA and DOX compared with the DOX group (Fig. 2A and B). Furthermore, the expression levels of TNF- $\alpha$ and IFN- $\gamma$ and the levels of ROS were significantly increased in DOX-treated H9c2 and AC16 cardiomyocytes compared with the control group, whereas treatment with C5aRA effectively reduced the DOX-induced effects (Fig. 2C-F). These results suggested that C5aRA may inhibit inflammation in DOX-induced cardiomyocytes.

C5aRA protects $H 9 c 2$ and ACl6 cardiomyocytes against DOX-induced senescence. To determine the roles of C5a and $\mathrm{C} 5 \mathrm{aR}$ in DOX-induced cardiomyocyte senescence, SA- $\beta$-gal staining was performed to assess cell aging. The treatment of H9c2 and AC16 cardiomyocytes with DOX significantly increased the percentage of senescent cells, while C5aRA treatment significantly reversed this effect (Fig. 3A and B). In addition, the protein expression levels of p53, p16, p21 and IGFBP3 were upregulated in DOX-treated H9c2 and AC16 cardiomyocytes compared with the control group, while 

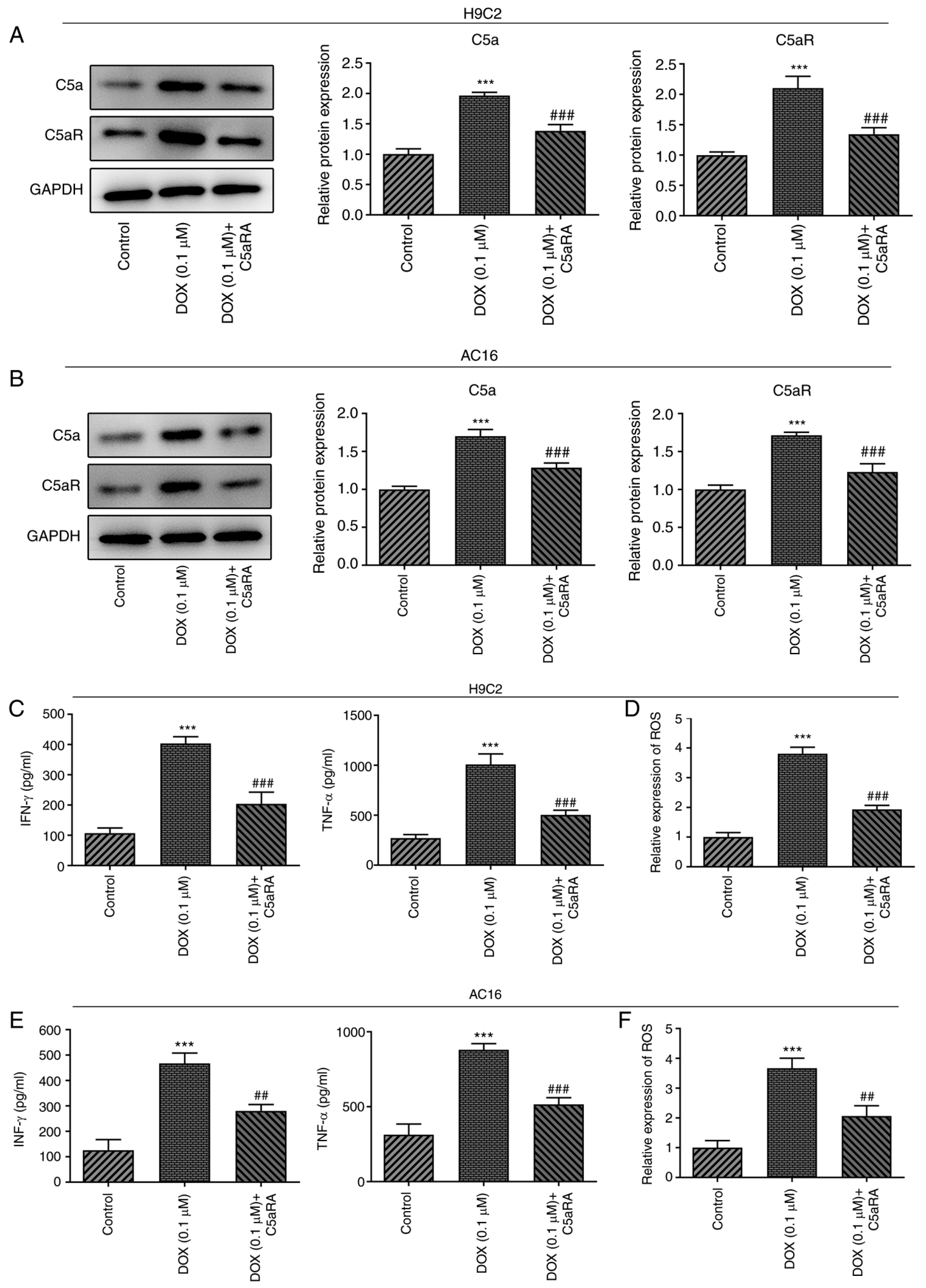

Figure 2. C5aRA inhibits inflammation in DOX-induced cardiomyocytes. (A and B) Expression levels of C5a and C5aR in H9c2 and AC16 cardiomyocytes as determined using reverse transcription-quantitative PCR analysis and western blotting. TNF- $\alpha$, IFN- $\gamma$ and ROS levels in (C and D) H9c2 and (E and F) AC16 cardiomyocytes were measured using ELISA. The results are representative of at least three independent experiments. ${ }^{* * *} \mathrm{P}<0.001$ vs. control group; ${ }^{* \#} \mathrm{P}<0.01$ and ${ }^{\# \#} \mathrm{P}<0.001$ vs. DOX group. C5aRA, complement component 5a receptor antagonist; C5a, complement component 5a; C5aR, complement component 5a receptor; DOX, doxorubicin; ROS, reactive oxygen species.

pretreatment with C5aRA antagonized the effects induced by DOX (Fig. 3C and D). These results suggested that C5aRA may protect $\mathrm{H} 9 \mathrm{c} 2$ and $\mathrm{AC} 16$ cardiomyocytes against DOX-induced senescence.
C5aRA inhibits the DOX-induced reduction in telomere length and telomerase activity in $\mathrm{H} 9 \mathrm{c} 2$ and AC16 cardiomyocytes. To further validate the role of C5a and C5aR in DOX-induced cardiomyocyte senescence, both the telomere length and 

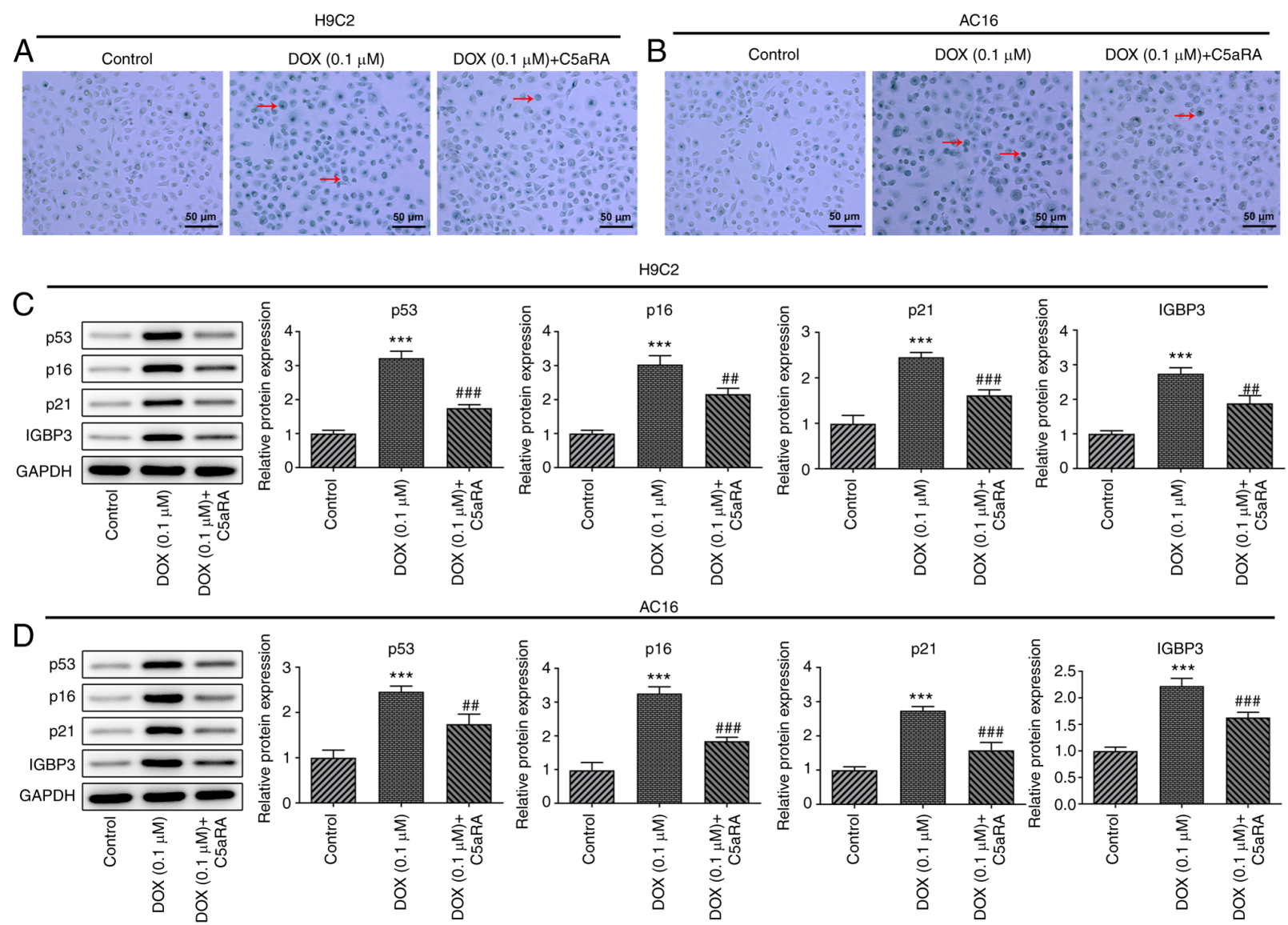

Figure 3. C5aRA protects $\mathrm{H} 9 \mathrm{c} 2$ and AC16 cardiomyocytes against DOX-induced senescence. (A and B) Representative images of the co-staining for SA- $\beta$-gal-positive cells. Magnification, x200. Scale bar, $50 \mu \mathrm{m}$. Arrows indicate positive cells. (C and D) Analysis of p53, p16, p21 and IGFBP3 protein expression levels. The results are representative of at least three independent experiments. ${ }^{* * *} \mathrm{P}<0.001$ vs. control group; ${ }^{\# \#} \mathrm{P}<0.01$ and ${ }^{\# \# \#} \mathrm{P}<0.001$ vs. DOX group. C5aRA, complement component 5a receptor antagonist; DOX, doxorubicin; SA- $\beta$-gal, senescence-associated $\beta$-galactosidase; IGFBP3, insulin-like growth factor binding protein 3 .

telomerase activity were measured in cardiomyocytes. Changes in telomere length and telomerase activity have been suggested to be potential biomarkers of aging (21). As shown in Fig. 4, C5aRA treatment markedly increased the telomere length and telomerase activity, whereas the telomerase activity was decreased in both H9c2 and AC16 cardiomyocytes following exposure to DOX. These results indicated that DOX treatment may induce telomere shortening and decrease telomerase activity, which may be alleviated by treatment with C5aRA.

\section{Discussion}

DOX is a widely used and potent anticancer agent; however, dose-dependent DOX-induced cardiotoxicity significantly limits its use in clinical practice (22). DOX was discovered to induce a senescent-like phenotype in cardiomyocytes and DOX-induced cytotoxicity may promote the progression of cellular senescence (23). Thus, there is great value in identifying effective therapies for suppressing cardiomyocyte senescence in order to prevent DOX-induced cardiotoxicity.

Components of the complement system have emerged as crucial mediators of tumor progression (24). Products of complement activation are known to maintain long-term inflammation, facilitate an immunosuppressive microenvironment, induce angiogenesis and enhance the migratory and metastatic potential of cancer cells (25). Recent in-depth studies on the role of $\mathrm{C} 5 \mathrm{a}$ in cancer have provided novel insight into the potential development of biomarkers and therapeutic approaches targeting C5a. C5a was found to play a crucial role in the suppression of joint and skin autoimmune inflammation mediated by neutrophils (26). The inhibition of $\mathrm{C} 5 \mathrm{aR}$ was also reported to be a substitute for the use of oral glucocorticoids, as C5a was identified to be an important inflammatory mediator in anti-neutrophil cytoplasm antibody-associated vascular inflammation (27). Thus, the role of C5a and C5aR in cardiomyocyte senescence was further investigated in the present study. The findings demonstrated that the expression levels of C5a and C5aR were upregulated in both $\mathrm{H} 9 \mathrm{c} 2$ and AC16 cardiomyocytes following DOX stimulation. Subsequently, the effects of C5a and C5aR on cardiomyocyte senescence were determined.

C5aRA blocks the binding of the C5a antitoxin with its receptor, $\mathrm{C} 5 \mathrm{aR}$, and was discovered to be one of the most effective agents in the treatment of various autoimmune diseases and acute inflammatory conditions $(28,29)$. A previous study suggested that inhibition of C5aR may hold promise as a therapeutic strategy for preventing organ injury in angiotensin II-induced hypertension (30). The results of the present study revealed that treatment of cardiomyocytes with C5aRA downregulated the expression levels of C5a and C5aR following 

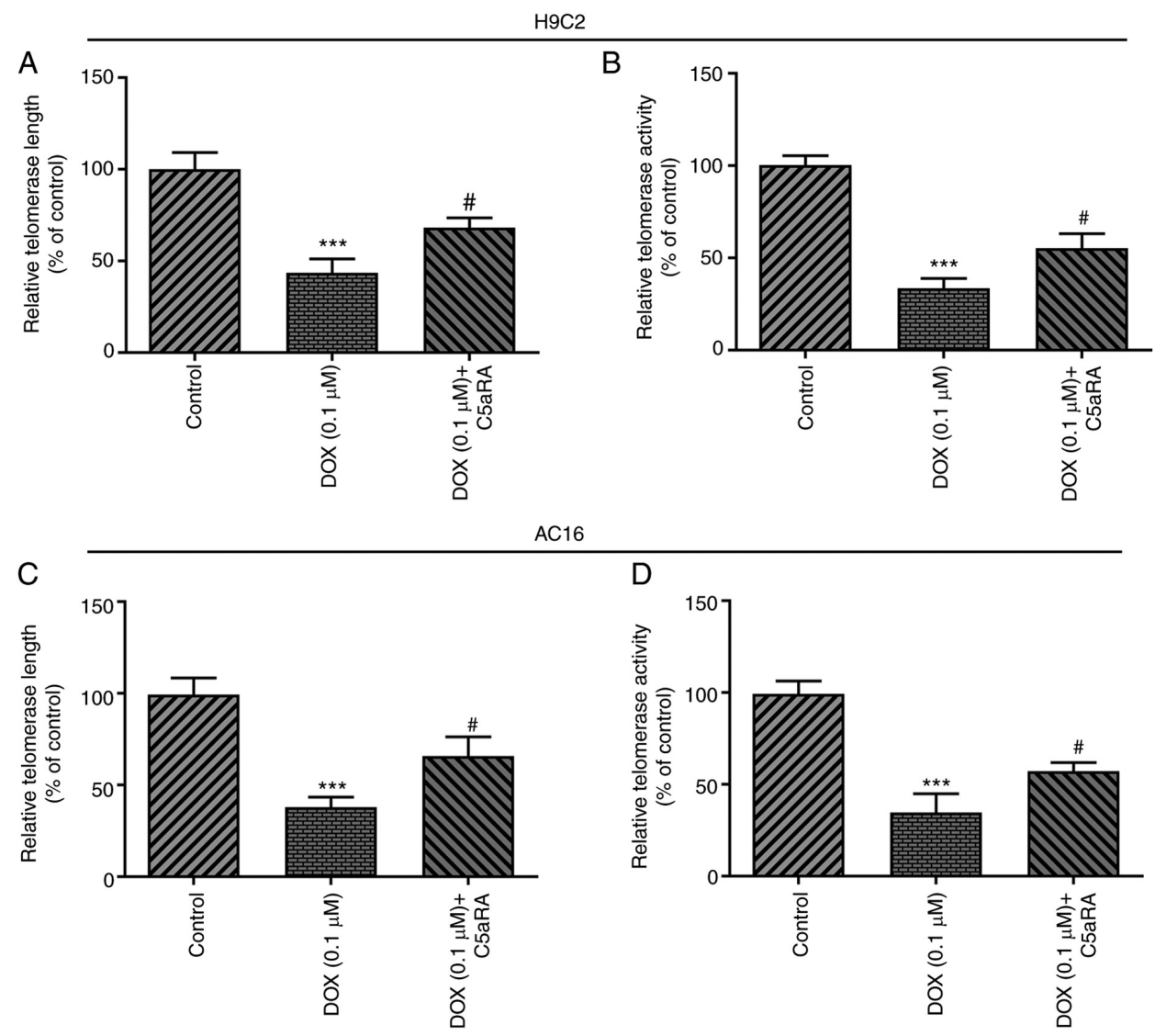

Figure 4. Treatment with C5aRA protects $\mathrm{H} 9 \mathrm{c} 2$ and AC16 cardiomyocytes against DOX-induced senescence. (A) Analysis of telomere length in H9c2 cardiomyocytes was performed using RT-qPCR. (B) Relative telomerase activity in H9c2 cardiomyocytes was measured using the TRAP assay. (C) Analysis of telomere length in AC16 cardiomyocytes was performed using RT-qPCR. (D) Relative telomerase activity in AC16 cardiomyocytes was measured using the TRAP assay. ${ }^{* * *} \mathrm{P}<0.05$ vs. control group; ${ }^{*} \mathrm{P}<0.05$ vs. DOX group. C5aRA, complement component 5a receptor antagonist; DOX, doxorubicin; RT-qPCR, reverse transcription-quantitative PCR; TRAP, telomeric repeat amplification protocol.

DOX treatment. In addition, the increased levels of inflammatory factors and ROS induced by DOX stimulation were also reduced following treatment with C5aRA.

The presence of low-grade inflammation has been recognized as a defining characteristic of aging (31). In the present study, two different characteristics of aging (telomere shortening and reduced telomerase activity) were further analyzed. The results demonstrated that DOX treatment upregulated the levels of SA- $\beta$-gal, the expression levels of the cell cycle inhibitor proteins, p53, p16 and p21, and the expression levels of IGFBP3. These effects were reversed following the co-culture with C5aRA. Telomere shortening and reduced telomerase activity in humans were also identified to be predictive markers of aging and disease (32-34). Thus, the telomere length and telomerase activity in cardiomyocytes were analyzed in the present study. The results revealed that treatment with Ca5RA suppressed the DOX-induced decrease in telomere length and telomerase activity in cardiomyocytes.

In conclusion, the findings of the present study revealed that the expression levels of C5a and C5aR were upregulated in DOX-induced cardiomyocytes, while treatment with C5aRA inhibited DOX-induced cardiomyocyte senescence. These findings suggest that C5a and C5aR may play a key role in DOX-induced cardiomyocyte senescence and provide a theoretical basis for future clinical applications. C5aRA treatment may be an effective method for protecting cardiomyocytes against DOX-induced senescence and may prove to be of value as a cardioprotective agent. However, a limitation of the present study is the lack of further validation in animal experiments and clinical samples, which will addressed in future studies.

\section{Acknowledgements}

Not applicable.

\section{Funding}

The present study was supported by the Key Discipline Construction Project of Shenzhen Municipal Health Commission (grant no. SZXJ2018007).

\section{Availability of data and materials}

The datasets used and/or analyzed during the current study are available from the corresponding author on reasonable request.

\section{Authors' contributions}

HW designed the research study. YW and FS performed the research and analyzed the data. FS wrote the main manuscript text. All authors contributed to revising the manuscript. All 
authors have read and approved the final manuscript. All authors confirm the authenticity of all the raw data.

\section{Ethics approval and consent to participate}

Not applicable.

\section{Patient consent for publication}

Not applicable.

\section{Competing interests}

The authors declare that they have no conflicts of interest.

\section{References}

1. Xu T, Ding W, Ji X, Ao X, Liu Y, Yu W and Wang J: Oxidative stress in cell death and cardiovascular diseases. Oxid Med Cel Longev 2019: 9030563, 2019.

2. Tang X, Li PH and Chen HZ: Cardiomyocyte senescence and cellular communications within myocardial microenvironments Front Endocrinol (Lausanne) 11: 280, 2020.

3. Hernandez-Segura A, Nehme $\mathbf{J}$ and Demaria M: Hallmarks of cellular senescence. Trends Cell Biol 28: 436-453, 2018.

4. Liu D, Ma Z, Di S, Yang Y, Yang J, Xu L, Reiter RJ, Qiao S and Yuan J: AMPK/PGCl $\alpha$ activation by melatonin attenuates acute doxorubicin cardiotoxicity via alleviating mitochondrial oxidative damage and apoptosis. Free Radic Biol Med 129: 59-72, 2018

5. Zhang YW, Shi J, Li YJ and Wei L: Cardiomyocyte death in doxorubicin-induced cardiotoxicity. Arch Immunol Ther Exp (Warsz) 57: 435-445, 2009

6. Singh P, Sharma R, McElhanon K, Allen CD, Megyesi JK, Beneš $\mathrm{H}$ and Singh SP: Sulforaphane protects the heart from doxorubicin-induced toxicity. Free Radic Biol Med 86: 90-101, 2015

7. Maejima Y, Adachi S, Ito H, Hirao K and Isobe M: Induction of premature senescence in cardiomyocytes by doxorubicin as a novel mechanism of myocardial damage. Aging Cell 7: 125-136, 2008.

8. Guo RF and Ward PA: Role of C5a in inflammatory responses. Annu Rev Immunol 23: 821-852, 2005.

9. Phieler J, Chung KJ, Chatzigeorgiou A, Klotzsche-von Ameln A, Garcia-Martin R, Sprott D, Moisidou M, Tzanavari T, Ludwig B, Baraban E, et al: The complement anaphylatoxin C5a receptor contributes to obese adipose tissue inflammation and insulin resistance. J Immunol 191: 4367-4374, 2013.

10. Jiang Y, Zhao G, Song N, Li P, Chen Y, Guo Y, Li J, Du L, Jiang S, Guo R, et al: Blockade of the C5a-C5aR axis alleviates lung damage in hDPP4-transgenic mice infected with MERS-CoV. Emerg Microbes Infect 7: 77, 2018.

11. Yu S, Wang D, Huang L, Zhang Y, Luo R, Adah D, Tang Y, Zhao K and Lu B: The complement receptor C5aR2 promotes protein kinase R expression and contributes to NLRP3 inflammasome activation and HMGB1 release from macrophages. J Biol Chem 294: 8384-8394, 2019.

12. Merle NS, Grunenwald A, Rajaratnam H, Gnemmi V, Frimat M, Figueres ML, Knockaert S, Bouzekri S, Charue D, Noe R, et al: Intravascular hemolysis activates complement via cell-free heme and heme-loaded microvesicles. JCI Insight 3: e96910, 2018.

13. Vlaicu SI, Tatomir A, Boodhoo D, Vesa S, Mircea PA and Rus H: The role of complement system in adipose tissue-related inflammation. Immunol Res 64: 653-664, 2016.

14. Martin IV, Bohner A, Boor P, Shagdarsuren E, Raffetseder U, Lammert F, Floege J, Ostendorf T and Weber SN: Complement C5a receptors C5L2 and C5aR in renal fibrosis. Am J Physiol Renal Physiol 314: F35-F46, 2018.

15. Brennan FH, Gordon R, Lao HW, Biggins PJ, Taylor SM, Franklin RJ, Woodruff TM and Ruitenberg MJ: The Complement receptor $\mathrm{C} 5 \mathrm{aR}$ controls acute inflammation and astrogliosis following spinal cord injury. J Neurosci 35: 6517-6531, 2015.

16. Leaker BR, Malkov VA, Mogg R, Ruddy MK, Nicholson GC, Tan AJ, Tribouley C, Chen G, De Lepeleire I, Calder NA, et al: The nasal mucosal late allergic reaction to grass pollen involves

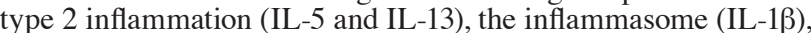
and complement. Mucosal Immunol 10: 408-420, 2017.
17. Yang B, Wang J, Zhao Y, Duan W, Dai C, Han Z, Wang M, Zhang B, Wei L, Chen Z and Chen D: Attenuating ischemia/reperfusion injury in rat cardiac transplantation by intracoronary infusion with siRNA cocktail solution. Biosci Rep 40: BSR20193937, 2020.

18. Zhang XL, Ji XT, Sun B, Qian LL, Hu XL, Lou HX and Yuan HQ: Anti-cancer effect of marchantin $\mathrm{C}$ via inducing lung cancer cellular senescence associated with less secretory phenotype. Biochim Biophys Acta Gen Subj 1863: 1443-1457, 2019.

19. Livak KJ and Schmittgen TD: Analysis of relative gene expression data using real-time quantitative PCR and the 2(-Delta Delta C(T)) method. Methods 25: 402-408, 2001.

20. Cawthon RM: Telomere length measurement by a novel monochrome multiplex quantitative PCR method. Nucleic Acids Res 37: e21, 2009.

21. Tedone E, Huang E, O'Hara R, Batten K, Ludlow AT, Lai TP, Arosio B, Mari D, Wright WE and Shay JW: Telomere length and telomerase activity in T cells are biomarkers of high-performing centenarians. Aging Cell 18: e12859, 2019.

22. Gupta SK, Garg A, Bär C, Chatterjee S, Foinquinos A, Milting H, Streckfuß-Bömeke K, Fiedler J and Thum T: Quaking inhibits doxorubicin-mediated cardiotoxicity through regulation of cardiac circular RNA expression. Circ Res 122: 246-254, 2018.

23. Huang PP, Fu J, Liu LH, Wu KF, Liu HX, Qi BM, Liu Y and Qi BL: Honokiol antagonizes doxorubicin-induced cardiomyocyte senescence by inhibiting TXNIP-mediated NLRP3 inflammasome activation. Int J Mol Med 45: 186-194, 2020.

24. Afshar-Kharghan V: The role of the complement system in cancer. J Clin Invest 127: 780-789, 2017.

25. Ajona D, Ortiz-Espinosa S and Pio R: Complement anaphylatoxins $\mathrm{C} 3 \mathrm{a}$ and $\mathrm{C} 5 \mathrm{a}$ : Emerging roles in cancer progression and treatment Semin Cell Dev Biol 85: 153-163, 2019.

26. Sadik CD, Miyabe Y, Sezin T and Luster AD: The critical role of $\mathrm{C} 5 \mathrm{a}$ as an initiator of neutrophil-mediated autoimmune inflammation of the joint and skin. Semin Immunol 37: 21-29, 2018.

27. Jayne DRW, Bruchfeld AN, Harper L, Schaier M, Venning MC, Hamilton P, Burst V, Grundmann F, Jadoul M, Szombati I, et al: Randomized trial of C5a receptor inhibitor avacopan in ANCA-associated vasculitis. J Am Soc Nephrol 28: 2756-2767, 2017.

28. Syed SN, Rau E, Ziegelmann M, Sogkas G, Brune B and Schmidt RE: C5aR activation in the absence of C5a: A new disease mechanism of autoimmune hemolytic anemia in mice. Eur J Immunol 48: 696-704, 2018.

29. Tang M, Zhang K, Li Y, He QH, Li GQ, Zheng QY and Zhang KQ: Mesenchymal stem cells alleviate acute kidney injury by down-regulating $\mathrm{C} 5 \mathrm{a} / \mathrm{C} 5 \mathrm{aR}$ pathway activation. Int Urol Nephrol 50: 1545-1553, 2018.

30. Zhang C, Li Y, Wang C, Wu Y and Du J: Antagonist of C5aR prevents cardiac remodeling in angiotensin II-induced hypertension. Am J Hypertens 27: 857-864, 2014.

31. Minguzzi M, Cetrullo S, D'Adamo S, Silvestri Y, Flamigni F and Borzi RM: Emerging players at the intersection of chondrocyte loss of maturational arrest, oxidative stress, senescence and low-grade inflammation in osteoarthritis. Oxid Med Cell Longev 2018: 3075293, 2018.

32. Ornish D, Lin J, Chan JM, Epel E, Kemp C, Weidner G, Marlin R, Frenda SJ, Magbanua MJM, Daubenmier J, et al: Effect of comprehensive lifestyle changes on telomerase activity and telomere length in men with biopsy-proven low-risk prostate cancer: 5-year follow-up of a descriptive pilot study. Lancet Oncol 14: 1112-1120, 2013.

33. Louzon M, Coeurdassier M, Gimbert F, Pauget B and de Vaufleury A: Telomere dynamic in humans and animals: Review and perspectives in environmental toxicology. Environ Int 131: 105025, 2019.

34. Victorelli S, Lagnado A, Halim J, Moore W, Talbot D, Barrett K, Chapman J, Birch J, Ogrodnik M, Meves A, et al: Senescent human melanocytes drive skin ageing via paracrine telomere dysfunction. EMBO J 38: e101982, 2019.

This work is licensed under a Creative Commons Attribution-NonCommercial-NoDerivatives 4.0 International (CC BY-NC-ND 4.0) License. 